\title{
- Inserana
}

Trascender, Contabilidad y Gestión Núm. 11 (mayo - agosto del 2019).

ISSN: 2448-6388. Universidad de Sonora. Departamento de Contabilidad.

Reserva de Derechos 04-2015-04172070800-203.

\section{Evolución de las Normas de Información Financiera en México}

\author{
Evolution of Financial Reporting Standards in Mexico
}

\begin{abstract}
María Eugenia De la Rosa Leal ${ }^{1}$; Mónica Ballesteros Grijalva ${ }^{2}$; Ismael Mario Gastélum Castro ${ }^{3}$
\end{abstract}
\section{Resumen}

Las Normas de Información Financiera, son criterios de comunicación y armonización de las prácticas contables en un contexto de globalización, cuyo objetivo es lograr entablar un lenguaje contable común en una convergencia internacional.

La profesión colegiada contable en México, a través del Instituto Mexicano de Contadores Públicos y el Consejo Mexicano de Normas de Información Financiera se ha preocupado en lograr esa concordancia, adaptando su teoría contable con y hacia las Normas Internacionales de Información Financiera.

Para hacerlo, ha transitado su marco contable en un camino de correcciones y adiciones de su anterior conceptualización de Principios de Contabilidad Generalmente Aceptados hacia Normas de Información Financiera en un cambio de enfoque del modelo contable de valuación, registro y presentación de los conceptos de los estados financieros.
Este trabajo presenta una revisión del acercamiento conceptual de las normas contables nacionales a un modelo normativo internacional, para lo cual se realizó un análisis de la evolución de las Normas de Información Financiera en México, como un acercamiento al modo de apropiación de conceptos e indicadores en la normatividad contable mexicana.

El trabajo inicia con la revisión conceptual de la normatividad contable, establece la revisión de la corriente normativa contable y sus características, llegando al marco conceptual normado.

Las conclusiones revisan la pertinencia del camino de adaptación del modelo normativo contable en México, con apertura hacia el valor razonable y revelación suficiente para organizaciones económicas.

Palabras clave: normatividad contable, desarrollo contable, México.

DOI: https://doi.org/10.36791/tcg.v11i0.63

1 María Eugenia De la Rosa Leal. Contador Público. Doctora en Ciencias Administrativas. Profesor investigador de la Academia de Contabilidad Superior del Departamento de Contabilidad de la Universidad de Sonora. Líder del cuerpo académico: Contabilidad, Gestión y Fiscalización. Correo: maedelarosa@hotmail.com

2 Mónica Ballesteros Grijalva. Contador Público. Doctora en Administración educativa. Presidente de la Academia de Contabilidad Superior del Departamento de Contabilidad de la Universidad de Sonora. Miembro del cuerpo académico: Contabilidad, Gestión y Fiscalización. Correo: monica.ballesteros@unison.mx

${ }^{3}$ Ismael Mario Gastélum Castro. Contador Público. Doctor en Administración educativa. Jefe del Departamento de Contabilidad de la Universidad de Sonora. Profesor investigador de la Academia de Auditoria. Miembro del cuerpo académico: Contabilidad, Gestión y Fiscalización. Correo: ismael.gastelum@unison.mx 


\section{Abstract}

The Financial Reporting Standards are criteria for communication and harmonization of accounting practices in a context of globalization, the objective of which is to achieve the inorder of a common accounting language in an international convergence.

The collegiate accounting profession in Mexico, through the Mexican Institute of Public Accountants and the Mexican Council of Financial Reporting Standards has been concerned with achieving this concordance, adapting its accounting theory with and towards International Standards Financial Information.

To do so, it has moved its accounting framework on a path of corrections and additions to its previous conceptualization of Generally Accepted Accounting Principles towards Financial Reporting Standards in a shift in focus on the valuation accounting model, registration and presentation of the concepts of the financial statements.
This work presents a review of the conceptual approach of national accounting standards to an international regulatory model, for which an analysis of the evolution of the Financial Reporting Standards in Mexico was carried out, as an approach to the way in which appropriation of concepts and indicators in Mexican accounting regulations.

The work begins with the conceptual review of accounting regulations, establishes the revision of the current accounting regulations and their characteristics, reaching the conceptual framework normative.

The conclusions review the relevance of the path to adapting the accounting regulatory model in Mexico, with openness to fair value and sufficient disclosure for economic organizations.

Keywords: accounting regulations, accounting development, Mexico.

JEL: M41 Contabilidad.

Recibido: 14 de febrero de 2019.

Aceptado: 14 de julio de 2019. 


\section{Introducción}

Los estados contables de una organización, revelan la situación económica y los resultados de la misma, permitiendo captar la imagen financiera actual, comparada y proyectada a distintos usuarios.

La credibilidad de los estados contables, ha sufrido desconfianza por algunas situaciones de fraude en la información, generadas con intención de confundir a los usuarios, y crear una imagen ficticia de éxito y prosperidad. Casos corporativos como Xerox (19972001); Enron (2001); WorlCom (2002); Olympus (2011), cuyas cifras contables se alteraron intencionalmente y fueron descubiertos, crearon un ambiente negativo de la contabilidad y los profesionales contables, por las estafas disfrazadas contablemente.

La situación anterior, más la globalización dominante en los mercados financieros ha estimulado el camino hacia la normalización y armonización contable en la generación de información, función adoptada por los organismos colegiados nacionales e internacionales, pactándose distintas fechas para lograr que los sistemas y modelos contables sean iguales, transformando los modelos nativos a un sistema armonizado. Siendo la última fecha pactada por la Federación Internacional de Contadores (IFAC) 2020.

Recordemos que un modelo como propuesta teórica contable, establece un patrón de fundamentos como esquema de una estructura compuesto por un sistema de criterios de la unidad monetaria, valuación de recursos, obligaciones y mantenimiento de capital como una propuesta de conceptos para el registro de la evolución patrimonial (Arguello, 2000).

En el modelo contable, la unidad monetaria puede ser: heterogénea nominal u homogénea; la valuación a costo histórico o valores corrientes y el capital a mantener: financiero: de enfoque en los propietarios y operativo; o de enfoque del ente (Arguello, 2000).
La formación de los modelos contables obedece a tres variables: económica, legal y contable, las cuales han dado lugar a dos modelos contables básicos: el modelo anglosajón y el modelo continental, cuya diferencia es el grado de desarrollo y complejidad de los registros y los negocios de la zona geográfica de uso.

Las Normas de Información Financiera (NIF), son un instrumento de armonización de las prácticas contables de registro, presentación y revelación, en un contexto de globalización, que genera un nuevo modelo contable: el modelo normativo -que combina a los modelos y suma preceptos generalizados-sujeto a disposiciones e interpretaciones preconcebidas, con dos espacios de decisión: el valor razonable y el juicio profesional.

Las NIF son de facto un motor de armonización de la práctica contable, cuyo objetivo es establecer mecanismos informativos, que a su vez monitorean las prácticas de registro y revelación de los conceptos de los estados financieros en razón de los escándalos contables (Martínez, Prado, \& Fernández, 2012). Así, el uso de las Normas de Información como estructura a seguir, busca recuperar la credibilidad contable.

En este esfuerzo de armonización, México se ha sumergido en una miscelánea normativa contable que cada año, propone, ausculta y emite normas contables de sus distintas series, desde una visión funcionalista en esencia. Definiendo a la contabilidad como:

"una técnica que se utiliza para el registro de operaciones que afectan económicamente a una entidad $y$ que produce sistemáticamente $y$ estructuradamente información financiera" (Instituto Mexicano de Contadores Públicos , 2019; NIF A1:5)

Esta definición tradicional, limita el alcance científico de la contabilidad, el cual trata de ser ampliado en las Normas A-3 y A-4, a partir de establecer al sistema contable, surgido a través de un reconocimiento contable 
y la presencia de requisitos de calidad y de complemento de información financiera y no financiera, a partir del cumplimiento de veracidad, objetividad y verificabilidad (Instituto Mexicano de Contadores Públicos , 2019: NIF A3, NIFA4).

Considerando lo anterior, este trabajo, se centra en estudiar, ¿hasta dónde la normalización contable y la búsqueda de la armonización normativa contable internacional se ha acercado a reflejar una imagen real?, implicando la asignación de valor a los recursos naturales tangibles e intangibles utilizados o afectados por la organización. Para lo que cual el marco de referencia utilizado son las Normas de Información Financiera mexicanas, dentro de la teoría del institucionalismo.

El trabajo presenta los antecedentes de las Normas de Información Financiera (NIF) en México, el análisis del marco conceptual, y de normas relacionadas de Conceptos Específicos. Relatando los resultados para concluir en los vacíos normativos y de comprensión como responsabilidad corporativa.

\section{Antecedentes}

Desde los años 70's el Instituto Mexicano de Contadores Públicos, en su calidad de organismo nacional de agrupación profesional contable, emitió los Principios de Contabilidad Generalmente Aceptados para orientar a los contadores públicos en el registro de los hechos económicos de las entidades y evitar que la información final al usuario pierda su utilidad y confiabilidad (Instituto Mexicano de Contadores Públicos , 2003: 11-13). En el mismo sentido a nivel internacional el Comité de Estándares Contables Internacionales (IASB. International Accounting Standards Bureau) emitió una serie de normas contables para que la información contable no pierda su utilidad y confiabilidad en los mercados internacionales, y represente fielmente la realidad económica de las empresas (Choi Frederick \& Mueller Gerhard, 1992:257-261).

El surgimiento de las Normas de Interpretación Financiera (NIF) en México, se da con un trasfondo de cambios económicos a nivel mundial y del país en particular. En el plano internacional, sin duda es la globalización de la economía la cual conlleva una mayor integración de las economías, a una mayor apertura de las mismas y a una mayor desregulación. Lo anterior impulsó una mayor armonización en diferentes normas contables de los diferentes países (Gracida, 2016).

Durante los ochenta y noventa del siglo XX, la normatividad contable fue emitida por la Comisión de Principios de Contabilidad Generalmente Aceptados (PCGA) del Instituto Mexicano de Contadores Públicos, A.C. (IMCP) a través de boletines y circulares, los que fueron evolucionando continuamente dependiendo de los cambios que sufrió el entorno de las empresas. En estos se establecieron los fundamentos de la contabilidad financiera en las que se baso las normas particulares y la respuesta a las controversias derivadas de la emisión de estados financieros de las entidades económicas. (CINIF,2013:p.xi citado por Gracida, 2016).

"Las Normas de Información Financiera evolucionan continuamente por cambios en el entorno y surgen como respuesta a las necesidades a las necesidades de los usuarios de la información contenida en los estados financieros y a las condiciones existentes. La globalización en el mundo de los negocios y de los mercados de capital está propiciando que la normatividad contable alrededor del mundo se armonice, teniendo como principal objetivo la generación de información financiera 
comparable, trasparente y de alta calidad, sobre el desempeño de las entidades económicas, que sirva a los objetivos de los usuarios generales." (Reyes s/f citado por Gracida, 2016).

A partir del 1 de junio de 2004, es el Consejo Mexicano para la Investigación y Desarrollo de Normas de Información Financiera, A.C. (CINIF) el encargado, según las tendencias mundiales, de la emisión de la normalidad contable en México, a través de un Consejo Emisor, integrado por investigadores de tiempo completo. El CINIF es el encargado directo de desarrollar dicha normatividad, después de procesos de investigación y auscultación, dando como resultado la emisión de documentos llamados "Normas de Información Financiera (NIF) (Gracida, 2016).

Las NIF comprenden las normas emitidas por el CINIF como los boletines emitidos por la Comisión de Principios de Contabilidad (CPC) que fueron transferidas al CINIF el 31 de mayo de 2004. Así el (CINIF) público a partir del 2005, cada año el libro de Normas de Información Financiera (NIF), el que tiene como objetivo congregar en un solo volumen, , todas las normas emitidas por el CINIF, así como aquéllas transferidas por la CPC del Instituto Mexicano de Contadores Públicos (IMCP); inciando su vigencia el primer grupo de NIF el 1 de enero del 2006. (NIF:2013, p.1569 citado por Gracida,2016).

En el año 2000, en razón de los sucesos fraudulentos de Enron, y World Com , se da énfasis a la transparencia informativa, involucrando a la contabilidad en la revelación de la gestión y el escrutinio de los resultados en una apertura comunitaria. Esperando de forma implícita que la información tenga la claridad y amplitud necesaria para conocer la calidad, hechos $\mathrm{y}$ consecuencias de los actos realizados.
El problema está, en ¿Cuáles son los indicadores y conceptos de la operación cotidiana de las organizaciones? Cuya respuesta puede ser, que dichos indicadores deben ser acotados a las posibilidades del sistema contable, mediante un estudio ontológico, con el supuesto general de que el control de las variables medioambientales de operación de la organización actúa favorablemente en la interiorización de las variables económicas.

El eje general de estudio, es la teoría de contabilidad de gestión, como un sistema al servicio de usuarios internos de la organización, cuyo propósito es utilizar los informes contables para la toma de decisiones, a la vez que permite medir la eficiencia de las acciones y decisiones tomadas por los administradores en sus actos de gestión, es de hecho una contabilidad directiva. Dando un sentido social a la gestión que se enfrenta cotidianamente a lo lógica económica del mejor negocio con el mayor rendimiento.

El proceso contable, garantiza la utilidad informativa por el cumplimiento de las características técnicas de confiabilidad: veracidad, objetividad y verificabilidad (Instituto Mexicano de Contadores Públicos, 2019: 14,22).

Aceptado el compromiso, el reto profesional contable, es lograr que la información final revele una imagen fiel, para lo cual lo primero es reconocer las limitaciones del sistema contable y las barreras teóricas para conceptualizar la teoría contable. Limitaciones, a las que se agrega el modelo contable nacional que establece un patrón de registro de eventos económicos que combina conceptos, criterios de reconocimiento y de asignación de valor, capturados en un sistema tecnológico para finalmente otorgar significación contable. 


\section{La Normas de Información Financiera en México}

En la actualidad las NIF en México, están compuestas por cinco series, 54 normas, 3 boletines de principios, 2 circulares, 7 interpretaciones, y 1 orientación de las normas de información financiera. Las series están clasificadas en Serie NIF A. Marco conceptual; Serie B. Normas aplicables a los estados financieros en su conjunto; Serie C. Normas aplicables a conceptos específicos de los estados financieros; Serie NIF D. Normas aplicables a problemas de determinación de resultados; y Serie NIF E. Normas aplicables a las actividades especializadas de distintos sectores.

El modelo contable en general, se integra para responder a un contexto macroeconómico y a la evolución de la doctrina contable en el país. En la cual, se considera a la fuente principal que suministra los fondos operativos, ya sean entidades financieras o el propio estado, separando la propiedad y dirección de las fuentes de financiamiento, lo cual conlleva a que sus usuarios sean principalmente inversores bursátiles y analistas financieros (De la Rosa \& Deniz , 2012).

Por otro lado, la influencia económico cultural afecta también en el diseño del modelo, así encontramos que:

"en los países latinos, con mercados financieros con un menor grado de desarrollo, existe una menor separación entre la propiedad y la dirección siendo el sector bancario una de las principales fuentes de financiación. En este contexto, la información contable ha tenido como principales destinatarios los propietarios actuales y los prestamistas, cuya principal preocupación ha sido el control de la gestión mediante la rendición de cuentas y la protección del patrimonio como garantía frente a los compromisos de pago contraídos con los acreedores” (De la Rosa \& Deniz , 2012: 188).

En México el modelo contable se identifica con el patrón continental cuyas características son: el alto grado de especialización y sofisticación en sus conceptos; la existencia de normas contables para un usuario general; regulación del ejercicio profesional por organismos representativos; y la generación de estados contables flexibles (De la Rosa, 2000: 11-16). Sumando la participación cada vez más activa de las regulaciones tributarias en la normatividad contable.

No perdamos, de vista que la contabilidad, es una más de un juego de herramientas administrativas de decisión, cuya estructura depende de la capacidad informativa, experiencia y preparación del cuerpo contable de la organización, lo cual la reviste y otorga un valor agregado y protagonismo en la información efectiva. Considerando que los estados contables no incluyen todos los eventos relevantes, por lo cual, son complementados o sustituidos con otros informes: de producción, de personal, de ventas, etc., que otorgan o suplen los vacíos de la información. Lo que en ocasiones provoca que los estados contables sean innecesarios o sustituibles por su incapacidad para representar la realidad económica completa de la empresa (De la Rosa, 1989: 65-68).

Haciendo un resumen de las influencias en el diseño del sistema contable sea normativo o de otra índole, estas se presentan en el cuadro 1. 
Cuadro 1. Variables de intervención en los modelos contables.

\begin{tabular}{|l|l|}
\hline \multicolumn{1}{|c|}{ Variable } & \multicolumn{1}{c|}{ Observación } \\
\hline Sistema legal & $\begin{array}{l}* \text { Ley común } \\
\text { *Derecho romano. Regulación gubernamental nacional y europea }\end{array}$ \\
\hline Fuentes de financiamiento & $\begin{array}{l}* \text { Sistema financiero nacional: bancos, financiadoras. } \\
\text { Terceros en relación de negocios: proveedores y acreedores en } \\
\text { general }\end{array}$ \\
\hline Tendencia contable & $\begin{array}{l}* \text { Enfoque de control, rendición de cuentas y predicción. } \\
\text { En razón de la naturaleza del negocio }\end{array}$ \\
\hline $\begin{array}{l}\text { Carácter público o privado de las } \\
\text { empresas }\end{array}$ & $\begin{array}{l}* \text { Valor ponderado de inversión en capital, e inversionistas } \\
\text { mayoritarios. }\end{array}$ \\
\hline Relación contabilidad-fiscalidad & $\begin{array}{l}* \text { Obligatoriedad o independencia de la contabilidad frente a las } \\
\text { disposiciones fiscales y su impacto en la base impositiva sobre el } \\
\text { resultado contable. }\end{array}$ \\
\hline Situación económica & $\begin{array}{l}* \text { Situación particular del país contra su balanza de pagos y desarrollo } \\
\text { interno. }\end{array}$ \\
\hline Nacionalismo político y económico & $\begin{array}{l}\text { Existencia de normas nacionales de actuación y relación global de } \\
\text { Estado en ellas. }\end{array}$ \\
\hline
\end{tabular}

Fuente: De la Rosa \& Deniz Mayor , 2012: 195. 
Cada una de estas variables impactan los criterios de emisión de las Normas de Información Financiera, resaltando la significación de conceptos contables y los aspectos protagónicos de gestión.

El contexto de las Normas de Información Financiera en México, de acuerdo a De la Rosa y Déniz (2012), el siguiente, vigente por más de diez años, sin cambios relevantes.

- Existe un organismo regulador y emisor de normas contables: CINIF (Consejo Mexicano de Investigación y Desarrollo de Normas de Información Financiera) con un consejo emisor compuesto por miembros de organismos empresariales, fiscalizadores, de educación superior, del sistema financiero, y de organismos colegiados ${ }^{1}$.

- Las autoridades fiscales emiten disposiciones tributarias que relacionan las cuentas y conceptos contables con la normatividad, como es el caso de las compras, las partidas monetarias y no monetarias, las obligaciones laborales, la facturación, etc.

- Todos los tipos de negocios del país se enmarcan en el Código de Comercio, la Ley General de Sociedades Mercantiles, y el grupo de leyes impositivas: Impuesto sobre la Renta, Impuesto sobre Valor Agregado y otros.

- Las disposiciones fiscales influyen en la auditoria de los estados financieros y en los cambios y adecuaciones de las normas contables.
- El alcance de observancia de las normas contables se circunscribe a empresas que cotizan en bolsa o tienen negocios internacionales. Siendo voluntario para el resto.

- Se acepta como medida de transición a los modelos contables internacionales el uso de la Norma supletoria que permite en uso de la normatividad internacional.

- La emisión y adaptación de las normas contables nacionales a las normas internacionales se encuentra en un período de modificaciones y cambios para lograr la transición internacional en el año 2020.

- Se vive un periodo intenso de educación normativa contable en el país, para convencer de sus beneficios de uso, a través de la estructura de colegios de contadores públicos afiliados al Instituto Mexicano de Contadores Públicos.

\section{Un estudio de la evolución normativa en México}

El objeto de estudio, de este trabajo fue la normatividad contable mexicana, para distinguir su evolución hacia el reconocimiento de información armonizada a revelar, para lo cual se aplicó un estudio documental de la cronología y características de la estructura de las Normas de Información Financiera (NIF), de 2006 a 2020, considerando que la vigencia de las normas conceptuales como punto central de las NIF fue a partir de 2006.

El interés de estudiar la ruta de tiempo y la tendencia de emisión de las Normas, tuvo como objetivo establecer el alcance conceptual de las Normas,

\footnotetext{
${ }^{1}$ La asamblea de asociados del CINIF se compone por la Asociación de Bancos de México, la Asociación de Intermediarios Bursátiles, la Asociación Mexicana de Instituciones de Seguros, la Asociación Nacional de Facultades y Escuelas de Contaduría y Administración, la Bolsa Mexicana de Valores, la Comisión Nacional Bancaria y de Valores, la Comisión Nacional de Seguros y Fianzas, el Consejo Coordinador Empresarial, el Consejo Mexicano de Hombres de Negocios, el Instituto Mexicano de Contadores Públicos, el Instituto Mexicano de Ejecutivos en Finanzas, la Secretaría de Hacienda y Crédito Público y la Secretaría de la Función Pública (CINIF, 2010: xi-xiv).
} 
estudiándose la emisión de las NIF en 2007, 2010, 2015, 2017 y 2019 presentado en las tablas 1, 2 y 3.
La metodología utilizada, fue el análisis semántico y taxonómico, de los conceptos normados.

Tabla 1. Análisis de la estructura de las Normas de Información Financiera en México. Resumen de emisiones y modificaciones (2006-2010).

\begin{tabular}{|l|c|c|c|c|c|c|}
\hline \multicolumn{1}{|c|}{ Normas } & $\begin{array}{c}\text { Antes } \\
2006\end{array}$ & 2006 & 2007 & 2008 & 2009 & 2010 \\
\hline Serie NIF A. Marco Conceptual. & & 8 & 0 & 0 & 0 & 0 \\
\hline $\begin{array}{l}\text { Serie NIF B. Normas aplicables a los estados } \\
\text { financieros en su conjunto. }\end{array}$ & 4 & 2 & 1 & 2 & 2 & 1 \\
\hline $\begin{array}{l}\text { Serie NIF C. Normas aplicables a conceptos } \\
\text { específicos de los estados financieros. }\end{array}$ & 10 & 0 & 1 & 0 & 2 & 1 \\
\hline $\begin{array}{l}\text { Serie NIF D. Normas aplicables a problemas } \\
\text { de determinación de resultados. }\end{array}$ & 2 & 0 & 1 & 2 & 1 & 0 \\
\hline $\begin{array}{l}\text { Serie NIF E. Normas aplicables a las } \\
\text { actividades especializadas de distintos } \\
\text { sectores. }\end{array}$ & 1 & 0 & 0 & 0 & 0 & 1 \\
\hline \hline Total & 17 & 10 & 3 & 4 & 5 & 3 \\
\hline
\end{tabular}

Tabla 1. Análisis de la estructura de las Normas de Información Financiera en México. Resumen de emisiones y modificaciones (2011-2015).

\begin{tabular}{|l|c|c|c|c|c|}
\hline \multicolumn{1}{|c|}{ Normas } & 2011 & 2012 & 2013 & 2014 & 2015 \\
\hline Serie NIF A. Marco Conceptual. & 0 & 0 & 0 & 0 & 0 \\
\hline $\begin{array}{l}\text { Serie NIF B. Normas aplicables a los estados } \\
\text { financieros en su conjunto. }\end{array}$ & 2 & 0 & 4 & 1 & 0 \\
\hline $\begin{array}{l}\text { Serie NIF C. Normas aplicables a conceptos } \\
\text { específicos de los estados financieros. }\end{array}$ & 4 & 0 & 2 & 3 & 0 \\
\hline $\begin{array}{l}\text { Serie NIF D. Normas aplicables a problemas de } \\
\text { determinación de resultados. }\end{array}$ & 0 & 0 & 0 & 0 & 0 \\
\hline $\begin{array}{l}\text { Serie NIF E. Normas aplicables a las actividades } \\
\text { especializadas de distintos sectores. }\end{array}$ & 0 & 0 & 0 & 0 & 0 \\
\hline \hline Total & 6 & 0 & 6 & 4 & 0 \\
\hline
\end{tabular}


Tabla 1. Análisis de la estructura de las Normas de Información Financiera en México. Resumen de emisiones y modificaciones (2016-2020).

\begin{tabular}{|c|c|c|c|c|c|c|}
\hline Normas & 2016 & 2017 & 2018 & 2019 & 2020 & Total \\
\hline Serie NIF A. Marco Conceptual. & 0 & 0 & 0 & 0 & 0 & 8 \\
\hline $\begin{array}{l}\text { Serie NIF B. Normas aplicables a los estados } \\
\text { financieros en su conjunto. }\end{array}$ & 0 & 0 & 1 & 0 & 1 & 17 \\
\hline $\begin{array}{l}\text { Serie NIF C. Normas aplicables a conceptos } \\
\text { específicos de los estados financieros. }\end{array}$ & 2 & 0 & 7 & 0 & 0 & 22 \\
\hline $\begin{array}{c}\text { Serie NIF D. Normas aplicables a problemas } \\
\text { de determinación de resultados. }\end{array}$ & 1 & 0 & 3 & 0 & 0 & 8 \\
\hline $\begin{array}{l}\text { Serie NIF E. Normas aplicables a las } \\
\text { actividades especializadas de distintos } \\
\text { sectores. }\end{array}$ & 0 & 0 & 0 & 0 & 1 & 2 \\
\hline Total & 3 & 0 & 11 & 0 & 2 & 57 \\
\hline
\end{tabular}

Fuente: Elaboración propia, análisis de información.

Como se observa en la tabla 1, la emisión y ampliación de las NIF se ha dirigido principalmente a dos series de armonización y regulación: la Serie C. Normas aplicables a Conceptos Específicos de los estados financieros (22 normas - 39\%), y la Serie B. Normas aplicables a los estados financieros en su conjunto (17 normas - 30 \%). las temáticas de las normas se presentan en la tabla 2 y 3. 
Tabla 2. Serie NIF B. Normas aplicables a los estados financieros en su conjunto.

\begin{tabular}{|c|c|c|c|c|}
\hline Norma & Antes 2006 & 2006 & 2007 & 2008 \\
\hline NIF B1 Cambios contables y correcciones de errores. & & vigente & & \\
\hline NIF B2 Estado de flujos de efectivo. & & vigente & & \\
\hline NIF B3 Estado de resultado integral. & & & vigente & \\
\hline NIF B4 Estado de cambios en capital contable. & $\begin{array}{l}\text { B4 Utilidad integral } \\
\text { i: } 2001 \text {. }\end{array}$ & & & \\
\hline NIF B5 Información financiera por segmentos. & $\begin{array}{l}\text { B5 Información } \\
\text { financiera por } \\
\text { segmentos i:2003. }\end{array}$ & & & \\
\hline \multicolumn{5}{|l|}{ NIF B6 Estado de Situación Financiera. } \\
\hline \multicolumn{5}{|l|}{ NIF B7 Adquisición de negocios. } \\
\hline \multicolumn{5}{|l|}{$\begin{array}{l}\text { NIF B8 Estados financieros consolidados o } \\
\text { combinados. }\end{array}$} \\
\hline NIF B9 Información financiera a fechas intermedias. & $\begin{array}{l}\text { B9 Información } \\
\text { financiera a fechas } \\
\text { intermedias i:1083. }\end{array}$ & & & \\
\hline NIF B10 Efectos de la inflación. & & & & vigente \\
\hline \multicolumn{5}{|l|}{$\begin{array}{l}\text { NIF B11 Disposición de activos de larga duración y } \\
\text { operaciones discontinuadas. }\end{array}$} \\
\hline \multicolumn{5}{|l|}{$\begin{array}{l}\text { NIF B12 Compensación de activos financieros y } \\
\text { pasivos financieros. }\end{array}$} \\
\hline \multirow[t]{2}{*}{$\begin{array}{l}\text { NIF B13 Hechos posteriores a la fecha de los estados } \\
\text { financieros. }\end{array}$} & & & vigente & \\
\hline & $\begin{array}{l}\text { B14 Utilidad por } \\
\text { acción i:1997. }\end{array}$ & & & \\
\hline NIF B15 Conversión de monedas extranjeras. & & & & vigente \\
\hline \multicolumn{5}{|l|}{$\begin{array}{l}\text { NIF B16 Estados financieros de entidades con } \\
\text { propósitos no lucrativos. }\end{array}$} \\
\hline \multicolumn{5}{|l|}{ NIF B17 Determinación del valor razonable. } \\
\hline Total & 4 & 2 & 1 & 2 \\
\hline
\end{tabular}


Tabla 2. Continuación...

\begin{tabular}{|c|c|c|c|c|}
\hline Norma & 2009 & 2010 & 2011 & 2012 \\
\hline \multicolumn{5}{|l|}{ NIF B1 Cambios contables y correcciones de errores. } \\
\hline \multicolumn{5}{|l|}{ NIF B2 Estado de flujos de efectivo. } \\
\hline \multicolumn{5}{|l|}{ NIF B3 Estado de resultado integral. } \\
\hline \multicolumn{5}{|l|}{ NIF B4 Estado de cambios en capital contable. } \\
\hline NIF B5 Información financiera por segmentos. & & & vigente & \\
\hline \multicolumn{5}{|l|}{ NIF B6 Estado de Situación Financiera. } \\
\hline NIF B7 Adquisición de negocios. & vigente & & & \\
\hline NIF B8 Estados financieros consolidados o combinados. & vigente & & & \\
\hline NIF B9 Información financiera a fechas intermedias. & & & vigente & \\
\hline \multicolumn{5}{|l|}{ NIF B10 Efectos de la inflación. } \\
\hline \multicolumn{5}{|l|}{$\begin{array}{l}\text { NIF B11 Disposición de activos de larga duración y } \\
\text { operaciones discontinuadas. }\end{array}$} \\
\hline \multicolumn{5}{|l|}{$\begin{array}{l}\text { NIF B12 Compensación de activos financieros y pasivos } \\
\text { financieros. }\end{array}$} \\
\hline \multicolumn{5}{|l|}{$\begin{array}{l}\text { NIF B13 Hechos posteriores a la fecha de los estados } \\
\text { financieros. }\end{array}$} \\
\hline \multicolumn{5}{|l|}{ NIF B15 Conversión de monedas extranjeras. } \\
\hline $\begin{array}{l}\text { NIF B16 Estados financieros de entidades con propósitos } \\
\text { no lucrativos. }\end{array}$ & & vigente & & \\
\hline \multicolumn{5}{|l|}{ NIF B17 Determinación del valor razonable. } \\
\hline Total & 2 & 1 & 2 & 0 \\
\hline
\end{tabular}


Tabla 2. Continuación...

\begin{tabular}{|c|c|c|c|c|}
\hline Norma & 2013 & 2014 & 2015 & 2016 \\
\hline \multicolumn{5}{|l|}{ NIF B1 Cambios contables y correcciones de errores. } \\
\hline \multicolumn{5}{|l|}{ NIF B2 Estado de flujos de efectivo. } \\
\hline NIF B3 Estado de resultado integral. & ampliada & & & \\
\hline NIF B4 Estado de cambios en capital contable. & vigente & & & \\
\hline \multicolumn{5}{|l|}{ NIF B5 Información financiera por segmentos. } \\
\hline NIF B6 Estado de Situación Financiera. & vigente & & & \\
\hline \multicolumn{5}{|l|}{ NIF B7 Adquisición de negocios. } \\
\hline NIF B8 Estados financieros consolidados o combinados. & ampliada & & & \\
\hline \multicolumn{5}{|l|}{ NIF B9 Información financiera a fechas intermedias. } \\
\hline \multicolumn{5}{|l|}{ NIF B10 Efectos de la inflación. } \\
\hline \multicolumn{5}{|l|}{$\begin{array}{l}\text { NIF B11 Disposición de activos de larga duración y } \\
\text { operaciones discontinuadas. }\end{array}$} \\
\hline $\begin{array}{l}\text { NIF B12 Compensación de activos financieros y pasivos } \\
\text { financieros. }\end{array}$ & & vigente & & \\
\hline \multicolumn{5}{|l|}{$\begin{array}{l}\text { NIF B13 Hechos posteriores a la fecha de los estados } \\
\text { financieros. }\end{array}$} \\
\hline \multicolumn{5}{|l|}{ NIF B15 Conversión de monedas extranjeras. } \\
\hline \multicolumn{5}{|l|}{$\begin{array}{l}\text { NIF B16 Estados financieros de entidades con propósitos no } \\
\text { lucrativos. }\end{array}$} \\
\hline \multicolumn{5}{|l|}{ NIF B17 Determinación del valor razonable. } \\
\hline Total & 4 & 1 & 0 & 0 \\
\hline
\end{tabular}


Tabla 2. Continuación...

\begin{tabular}{|c|c|c|c|c|}
\hline Norma & 2017 & 2018 & 2019 & 2020 \\
\hline \multicolumn{5}{|c|}{ NIF B1 Cambios contables y correcciones de errores. } \\
\hline \multicolumn{5}{|l|}{ NIF B2 Estado de flujos de efectivo. } \\
\hline \multicolumn{5}{|l|}{ NIF B3 Estado de resultado integral. } \\
\hline \multicolumn{5}{|l|}{ NIF B4 Estado de cambios en capital contable. } \\
\hline \multicolumn{5}{|l|}{ NIF B5 Información financiera por segmentos. } \\
\hline \multicolumn{5}{|l|}{ NIF B6 Estado de Situación Financiera. } \\
\hline \multicolumn{5}{|l|}{ NIF B7 Adquisición de negocios. } \\
\hline \multicolumn{5}{|c|}{ NIF B8 Estados financieros consolidados o combinados. } \\
\hline \multicolumn{5}{|c|}{ NIF B9 Información financiera a fechas intermedias. } \\
\hline \multicolumn{5}{|l|}{ NIF B10 Efectos de la inflación. } \\
\hline $\begin{array}{l}\text { NIF B11 Disposición de activos de larga dura } \\
\text { operaciones discontinuadas. }\end{array}$ & & & & vigente \\
\hline \multicolumn{5}{|c|}{$\begin{array}{l}\text { NIF B12 Compensación de activos financieros y pasivos } \\
\text { financieros. }\end{array}$} \\
\hline \multicolumn{5}{|c|}{$\begin{array}{l}\text { NIF B13 Hechos posteriores a la fecha de los estados } \\
\text { financieros. }\end{array}$} \\
\hline \multicolumn{5}{|l|}{ NIF B15 Conversión de monedas extranjeras. } \\
\hline \multicolumn{5}{|c|}{$\begin{array}{l}\text { NIF B16 Estados financieros de entidades con propósitos no } \\
\text { lucrativos. }\end{array}$} \\
\hline NIF B17 Determinación del valor razonable. & & vigente & & \\
\hline Total & 0 & 1 & 0 & 1 \\
\hline
\end{tabular}

Fuente: Elaboración propia, análisis de información. 
En la tabla 2, se observan los cambios y emisiones de las NIF en materia de estados financieros, privilegiando la atención temprana del Estado de flujo de efectivo y el Estado de Resultados integral, para lo cual se adecuaron normas para valuar y revelar activos financieros. Estableciendo recientemente - 2018- la norma de determinación de valor razonable o fair value.

Tabla 3. Serie NIF C. Normas aplicables a conceptos específicos de los estados financieros.

\begin{tabular}{|c|c|c|c|c|}
\hline Norma & Antes 2006 & 2006 & 2007 & 2008 \\
\hline \multicolumn{5}{|l|}{$\begin{array}{l}\text { NIF C1 Efectivo y equivalentes de } \\
\text { efectivo. }\end{array}$} \\
\hline $\begin{array}{l}\text { NIF C2 Inversión en instrumentos } \\
\text { financieros. }\end{array}$ & $\begin{array}{l}\text { C2 Instrumentos financieros i:2001; } \\
\text { m:2005. }\end{array}$ & & & \\
\hline NIF C3 Cuentas por cobrar. & C3 Cuentas por cobrar i:1974. & & & \\
\hline NIF C4 Inventarios. & C4 Inventarios i:1874. & & & \\
\hline NIF C5 Pagos anticipados. & C5 Pagos anticipados i:1981. & & & \\
\hline NIF C6 Propiedades, planta y equipo. & $\begin{array}{l}\text { C6 Inmuebles, maquinaria y equipo } \\
\text { i:1974. }\end{array}$ & & & \\
\hline \multicolumn{5}{|l|}{$\begin{array}{l}\text { NIF C7 Inversiones en asociadas, } \\
\text { negocios conjuntos y otras inversiones } \\
\text { permanentes. }\end{array}$} \\
\hline \multicolumn{5}{|l|}{ NIF C8 Activos intangibles. } \\
\hline $\begin{array}{l}\text { NIF C9 Provisiones, contingencias y } \\
\text { compromisos. }\end{array}$ & $\begin{array}{l}\text { C9 Pasivo, provisiones, activos y } \\
\text { pasivos contingentes y compromisos } \\
\mathrm{i}: 2003 \text {. }\end{array}$ & & & \\
\hline $\begin{array}{l}\text { NIF C10 Instrumentos derivados y } \\
\text { relaciones de cobertura. }\end{array}$ & $\begin{array}{l}\text { C10 Instrumentos financieros } \\
\text { derivados y operaciones de cobertura } \\
\mathrm{i}: 2005 \text {. }\end{array}$ & & & \\
\hline NIF C11 Capital Contable. & C11 Capital contable i:1990. & & & \\
\hline $\begin{array}{l}\text { NIF C12 Instrumentos financieros con } \\
\text { características de pasivo y capital. }\end{array}$ & $\begin{array}{l}\text { C12 Instrumentos financieros con } \\
\text { características de pasivo, de capital o } \\
\text { de ambos i:2004. }\end{array}$ & & & \\
\hline NIF C13 Partes relacionadas. & & & vigente & \\
\hline
\end{tabular}


Tabla 3. Continuación...

\begin{tabular}{|l|l|l|l|l|}
\hline \multicolumn{1}{|c|}{ Norma } & \multicolumn{1}{|c|}{ Antes 2006 } & 2006 & 2007 & 2008 \\
\hline & $\begin{array}{l}\text { C15 Deterioro en el valor de los } \\
\text { activos de larga duración y su } \\
\text { disposición i:2004. }\end{array}$ & & & \\
\hline $\begin{array}{l}\text { NIF C16 Deterioro de instrumentos } \\
\text { financieros por cobrar. }\end{array}$ & & & & \\
\hline $\begin{array}{l}\text { NIF C18 Obligaciones asociadas con el } \\
\text { retiro de propiedades, planta y equipo. }\end{array}$ & & & & \\
\hline $\begin{array}{l}\text { NIF C19 Instrumentos financieros por } \\
\text { pagar. }\end{array}$ & & & & \\
\hline $\begin{array}{l}\text { NIF C20 Instrumentos de } \\
\text { financiamiento por cobrar principal e } \\
\text { intereses. }\end{array}$ & & 10 & 0 & 1 \\
\hline $\begin{array}{l}\text { NIF C21 Acuerdos con control } \\
\text { conjunto. }\end{array}$ & & & & 0 \\
\hline \hline Total & & & & \\
\hline
\end{tabular}


Tabla 3. Continuación...

\begin{tabular}{|l|l|l|l|l|l|}
\hline \multicolumn{1}{|c|}{ Norma } & 2009 & 2010 & 2011 & 2012 & 2013 \\
\hline NIF C1 Efectivo y equivalentes de efectivo. & & vigente & & & \\
\hline $\begin{array}{l}\text { NIF C2 Inversión en instrumentos } \\
\text { financieros. }\end{array}$ & & & & & \\
\hline NIF C3 Cuentas por cobrar. & & & & & \\
\hline NIF C4 Inventarios. & & & vigente & & \\
\hline NIF C5 Pagos anticipados. & & & vigente & & \\
\hline NIF C6 Propiedades, planta y equipo. & vigente & & & & \\
\hline $\begin{array}{l}\text { NIF C7 Inversiones en asociadas, negocios } \\
\text { conjuntos y otras inversiones permanentes. }\end{array}$ & & & & & \\
\hline NIF C8 Activos intangibles. & & & & & \\
\hline $\begin{array}{l}\text { NIF C9 Provisiones, contingencias y } \\
\text { compromisos. }\end{array}$ & & & & & \\
\hline $\begin{array}{l}\text { NIF C10 Instrumentos derivados y } \\
\text { relaciones de cobertura. }\end{array}$ & & & & & \\
\hline $\begin{array}{l}\text { NIF C11 Capital Contable. } \\
\text { NIF C12 Instrumentos financieros con }\end{array}$ & & & & & \\
\hline
\end{tabular}


Tabla 3. Continuación...

\begin{tabular}{|l|l|l|l|l|l|}
\hline \multicolumn{1}{|c|}{ Norma } & 2009 & 2010 & 2011 & 2012 & 2013 \\
\hline NIF C13 Partes relacionadas. & & & & \\
\hline $\begin{array}{l}\text { NIF C14 Transferencia y baja de activos } \\
\text { financieros. }\end{array}$ & & & & & \\
\hline & & & & & \\
\hline $\begin{array}{l}\text { NIF C16 Deterioro de instrumentos } \\
\text { financieros por cobrar. }\end{array}$ & & & & & \\
\hline $\begin{array}{l}\text { NIF C18 Obligaciones asociadas con el } \\
\text { retiro de propiedades, planta y equipo. }\end{array}$ & & & & & \\
\hline $\begin{array}{l}\text { NIF C19 Instrumentos financieros por } \\
\text { pagar. }\end{array}$ & & & & & \\
\hline $\begin{array}{l}\text { NIF C20 Instrumentos de financiamiento } \\
\text { por cobrar principal e intereses. }\end{array}$ & & & & & \\
\hline NIF C21 Acuerdos con control conjunto. & & & & & \\
\hline \hline Total & & & & & \\
\hline
\end{tabular}


Tabla 3. Continuación...

\begin{tabular}{|c|c|c|c|c|c|c|}
\hline Norma & 2014 & 2015 & 2016 & 2017 & 2018 & 2019 \\
\hline \multicolumn{7}{|l|}{$\begin{array}{l}\text { NIF C1 Efectivo y equivalentes de } \\
\text { efectivo. }\end{array}$} \\
\hline $\begin{array}{l}\text { NIF C2 Inversión en instrumentos } \\
\text { financieros. }\end{array}$ & & & & & vigente & \\
\hline NIF C3 Cuentas por cobrar. & & & vigente & & ampliada & \\
\hline \multicolumn{7}{|l|}{ NIF C4 Inventarios. } \\
\hline \multicolumn{7}{|l|}{ NIF C5 Pagos anticipados. } \\
\hline \multicolumn{7}{|l|}{ NIF C6 Propiedades, planta y equipo. } \\
\hline \multicolumn{7}{|l|}{$\begin{array}{l}\text { NIF C7 Inversiones en asociadas, } \\
\text { negocios conjuntos y otras inversiones } \\
\text { permanentes. }\end{array}$} \\
\hline \multicolumn{7}{|l|}{ NIF C8 Activos intangibles. } \\
\hline $\begin{array}{l}\text { NIF C9 Provisiones, contingencias y } \\
\text { compromisos. }\end{array}$ & & & & & vigente & \\
\hline $\begin{array}{l}\text { NIF C10 Instrumentos derivados y } \\
\text { relaciones de cobertura. }\end{array}$ & & & & & vigente & \\
\hline NIF C11 Capital Contable. & vigente & & & & & \\
\hline $\begin{array}{l}\text { NIF C12 Instrumentos financieros con } \\
\text { características de pasivo y capital. }\end{array}$ & vigente & & & & & \\
\hline
\end{tabular}


Tabla 3. Continuación...

\begin{tabular}{|c|c|c|c|c|c|c|}
\hline Norma & 2014 & 2015 & 2016 & 2017 & 2018 & 2019 \\
\hline \multicolumn{7}{|l|}{ NIF C13 Partes relacionadas. } \\
\hline \multicolumn{7}{|l|}{$\begin{array}{l}\text { NIF C14 Transferencia y baja de activos } \\
\text { financieros. }\end{array}$} \\
\hline $\begin{array}{l}\text { NIF C16 Deterioro de instrumentos } \\
\text { financieros por cobrar. }\end{array}$ & & & & & vigente & \\
\hline \multicolumn{7}{|l|}{$\begin{array}{l}\text { NIF C18 Obligaciones asociadas con el } \\
\text { retiro de propiedades, planta y equipo. }\end{array}$} \\
\hline $\begin{array}{l}\text { NIF C19 Instrumentos financieros por } \\
\text { pagar. }\end{array}$ & & & & & vigente & \\
\hline $\begin{array}{l}\text { NIF C20 Instrumentos de financiamiento } \\
\text { por cobrar principal e intereses. }\end{array}$ & & & vigente & & ampliada & \\
\hline \multicolumn{7}{|l|}{ NIF C21 Acuerdos con control conjunto. } \\
\hline Total & 3 & 0 & 2 & 0 & 7 & 0 \\
\hline
\end{tabular}

Fuente: Elaboración propia, análisis de información. 
En la tabla 3, se observa como en relación con los Conceptos específicos, se han emitido lentamente distintas normas, como prueba y error, entre los cuales:

a) Se replanteó el boletín de instrumentos financieros, hasta emitir la NIF correspondiente.

b) Al igual ha sucedido con la norma correspondiente a pasivos y compromisos;

c) La norma de cuentas por cobrar ha sido revisada y emitida en dos ocasiones,

d) La norma de instrumentos de financiamiento por cobrar, e inversiones asociadas, se ha emitido en dos ocasiones.

En esta serie se han emitido propuestas de normas que no existían en los Principios de Contabilidad, el énfasis ha sido hacia los instrumentos financieros, encontrándose en auscultación la norma de cyber moneda.

El resumen de las emisiones y modificaciones de las NIF de 2006 a 2020 -periodo de vigencia-, se ilustra en los Gráficos 1 y 2.

Gráfico 1. Total de Normas de Información Financiera emitidas.

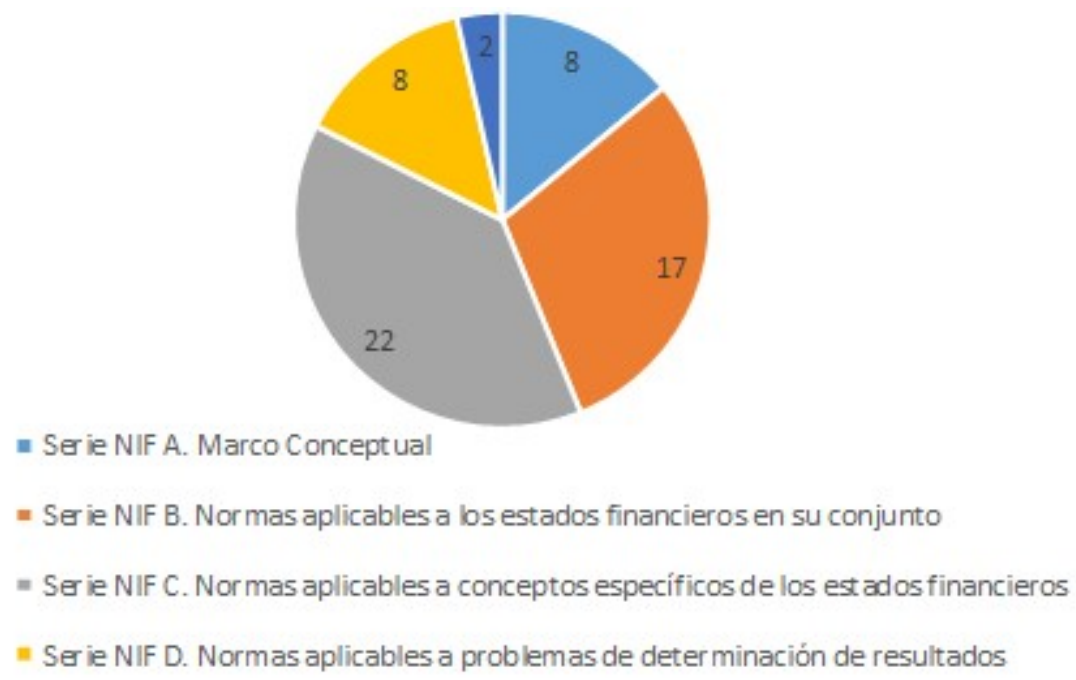

Fuente: Elaboración propia, análisis de la información. 
Dentro de las series la de menor cambios es la serie D, y la A. Esta última es con la que se inició la adopción de las NIF, manteniéndose sin cambios desde 2006, con la jurisdicción general sobre el resto de las series en su interpretación, asignación de valor, presentación y revelación.

Se observa que una norma conceptual es emitida, como particular en las normas serie B. Estados Financieros. La relativa a Determinación del valor razonable cuya conexión es directa con la Norma A6.
Reconocimiento y valuación. Existe un documento de Mejoras a las Normas de Información Financiera que justifica y explica las normas que generan cambios contables, como apoyo complementario, el cual se ha ido alimentando en la medida que el consejo emisor de las normas desarrolla precisiones de aplicación.

En el gráfico siguiente se presenta la tendencia de emisiones y modificaciones que a nivel conceptual han tenido las normas.

Gráfico 2. Normas de Información Financiera mexicanas. Tendencia de emisiones y modificaciones

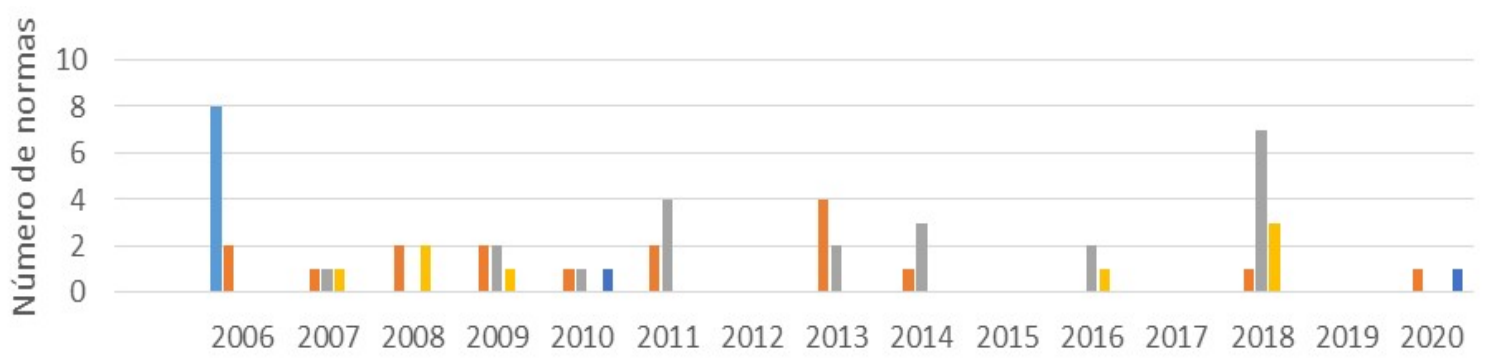

Año

- Serie NIF A. Marco Conceptual

- Serie NIF B. Normas aplicables a los estados financieros en su conjunto

- Serie NIF C. Normas aplicables a conceptos específicos de los estados financieros

- Serie NIF D. Normas aplicables a problemas de determinación de resultados

- Serie NIF E. Normas aplicables a las actividades especializadas de distintos sectores

Fuente: Elaboración propia, análisis de información.

El gráfico anterior, permite apreciar el impacto de normas NIF emitidas en México, existiendo normas con vigencia inicial en 2020.

Los ciclos de mayor cantidad de cambios normativos han sido 2006 y 2018, teniendo un trabajo constante y continuo en 2011 y 2014, mientras en 2013 y 2016 se trabajó principalmente en ampliaciones de Normas C. aplicables a estados financieros, no logrando a la fecha la armonización de todas las series de las NIF mexicanas, existiendo en la actualidad aún boletines de los Principios de Contabilidad por armonizar, como se observa en las tablas 1 y 2. 
Se observa, que la adaptación del modelo contable normativo de México al internacional, tiene una respuesta reactiva, atendiendo en primer orden los activos y pasivos financieros, provenientes de o para financiamientos. Atendiéndose de manera emergente las situaciones a través de Interpretaciones u Orientaciones de las Normas de Información Financiera emitidas como facultad del Consejo Mexicano de Normas de Información Financiera.

Las relaciones conceptuales, entre las normas analizadas presentaron la siguiente frecuencia, tabla 4.

Tabla 4. Frecuencia conceptual con las variables de estudio

\begin{tabular}{|c|l|l|c|}
\hline \multirow{2}{*}{ Variables } & \multicolumn{1}{|c|}{ Relación } & Concepto & $\begin{array}{c}\text { Frecuencia } \\
\text { en las NIF }\end{array}$ \\
\hline \multirow{2}{*}{$\begin{array}{c}\text { Normas de información } \\
\text { financiera }\end{array}$} & Son propiedad de & $\begin{array}{l}\text { La transparencia y la } \\
\text { revelación }\end{array}$ & 8 \\
\cline { 2 - 4 } & $\begin{array}{l}\text { Está asociada } \\
\text { con }\end{array}$ & $\begin{array}{l}\text { Contabilidad de Gestión } \\
\text { y/o Financiera }\end{array}$ & 15 \\
\hline & $\begin{array}{l}\text { Está asociada } \\
\text { con }\end{array}$ & $\begin{array}{l}\text { Tecnología y Acceso a la } \\
\text { información }\end{array}$ & 2 \\
\hline
\end{tabular}

Fuente: Elaboración propia, análisis de información.

La frecuencia se midió por la expresión textual de conceptos para entidades que emiten estados financieros en términos de las NIF. Encontrándose párrafos o conceptos que sitúan con claridad el alcance y características de la revelación para el usuario general sin que se exprese la posibilidad de reconocimiento de conceptos intangibles o sociales. Existiendo un apéndice de la NIF A3 que habla de otra información no financiera, sin embargo, no se otorga reconocimiento contable a esta información.

\section{Conclusiones}

1. Las Normas de Información Financiera se han vuelto en un modelo de observación obligado por las instituciones colegiadas contables en pro de la armonización internacional.

2. En México, se optó por un proceso de adaptación y evolución de sus Principios de Contabilidad Generalmente Aceptados como modelo nacional al de Normas de Información Financiera, lo cual ha significado replantear constantemente conceptos y reglas de reconocimiento contable, creando vacíos de aplicación en el país.

3. Los estados contables reflejan el grado de profesionalismo de la profesión contable en los países, en los cuales el modelo normativo contable ha tomado un papel protagónico sobre los demás modelos. 
4. La definición de la contabilidad como una técnica la limita y circunscribe a un alcance limitado de aspectos contingentes e intangibles, como el caso del medio ambiente y la responsabilidad social.

5. La información contable, es un elemento global de competitividad y calidad. Que logra comunicar una imagen pública.

6. En México la adecuación de las Normas de Información Financiera lleva trece años en los cuales ha sido arduo y sostenido el desarrollo de las mismas, viviendo una constante actualización y cambios en las reglas de reconocimiento, valuación y presentación sumados a los cambios de la política económica nacional.

7. La evolución normativa de las NIF en México se ha dirigido a establecer un marco conceptual, a partir del cual se han revisado y replanteado los cuatro estados financieros básicos, ampliando el catálogo de conceptos de registro y sus normas.

8. Un esfuerzo constante en México, ha sido la protección de la identidad contable, modernizando y respondiendo a la armonización internacional, habiendo aplazado en dos períodos su período de homologación total, a través de periodos de transición.

9. Una vez estudiado el modelo normativo actual de las NIF en México, se detectó una posibilidad de apertura conceptual, que no es claramente identificada para una evolución de una contabilidad científica. Pero sí para la apertura global a mercados financieros.

\section{Referencias:}

Arguello, G. (2000). Apuntes de Cátedra. Obtenido de Resumen-Modelos-Cotnables-Teoría: https://es.scribd.com/document/261591729/ResumenModelos-Contable.Teoría

Choi Frederick, D. S., \& Mueller Gerhard, G. (1992). International Accounting. New Jersey: Prentice Hall.

De la Rosa Leal , M. E., \& Deniz Mayor , J. J. (2012). Los modelos y sistemas contables nacionales . Los casos de México y España. En M. E. De la Rosa Leal , Análisis y aplicación de las Normas de Información Financiera (págs. 185-220). Hermosillo : Universidad de Sonora, Instituto Mexicano de Contadores Públicos Sonora .

De la Rosa-Leal, M. E. (2009). Modelo de costos medioambientales contables. Caso industria maquiladora. México : Plaza y Valdés.

Gracida Romo, J. J. (2016). Historia Económica de las NIF en Mexico. En M. E. De la Rosa Leal , Aplicación de las Normas de Información Financiera, Serie C, Conceptos específicos (págs. 19-26). Hermosillo, Sonora: Universidad de Sonora .

Instituto Mexicano de Contadores Públicos . (2003). Principios de Contabildad Generalmente Aceptados . México: IMCP.

Instituto Mexicano de Contadores Públicos . (2019). Normas de Información Financiera . México: IMCP.

Martínez Ferrero , J., Prado Lorenzo, J. M., \& Fernández Fernández, J. M. (2012). Responsabilidad Social Corporativa vs Responsabilidad Contable . Spanish Accounting Review, 32-45.

Vargas Hernández, J. G. (2005). Análisis de fundamentos de la teoría institucional . Revista Digital Universitaria , 2-21. 